\title{
Analysis of Uses of Technology in the Teaching of Mathematics
}

\author{
Morten Misfeldt and Wei-Chi Yang
}

\section{Overviews}

This Topic Study Group aimed at providing a forum to discuss the current state of art of the presence of technology in diverse aspects of teaching mathematics conveying a deep analysis of its implications to the future. Technology was understood in a broad sense, encompassing the computers of all types including the hand-held technology, the software of all types, and the technology of communication that includes the electronic board and the Internet. The discussions served as opportunity for all interested in the use of technology in education environment, to understand its diverse aspects and to share the creative and outstanding contributions, with critical analysis of the different uses.

The Topic Study Group had 42 contributions and more than 80 participants. The topics addressed were diverse but evolve around the use of technology in the classroom practice, design and use of digital teaching materials, Technology in teacher education, Distance education and the use of learning management systems. The use of technology in the teaching of mathematics is an expanding and diverse field, and in the following we will summarize the status and consensus that became apparent through the work in the Topic Study Group. One way to gain an overview of the image of the field presented in the topic study group is to look at the different

Organizers Co-chairs: Morten Misfeldt (Denmark), Wei-Chi Yang (USA); Team Members: Erol Karakirik (Turkey), Ngan Hoe Lee (Singapore), Cheong Soo Cho (Korea), Matte Andersen (Norway); Liaison IPC Menber: Yuriko Baldin (Brazil).

M. Misfeldt ( $\square)$

Aarhus University, Aarhus, Denmark

e-mail: mmi@dpu.dk

W.-C. Yang

Radford University, Radford, USA

e-mail: wyang@ radford.edu

(C) The Author(s) 2015 
technologies involved. The technologies adopted and described in the TSG did mainly fall into 6 categories (1) handheld and pc based computer algebra systems, (2) dynamic geometry systems, (3) learning management systems and internet access, (4) domain specific visualizations and manipulatives, (5) video streaming, and (6) touch technology such as ipads and smartboards.

Another way to gain such overview is to present the discussions and concerns that were prevalent in the discussion and contributions. These concerns relate to (a) an increase in efficiency of mathematics instruction with the aid of technologyincluding technological support for development of specific mathematical concepts and competencies, (b) teacher training and teacher practice with technology, (c) the use of technology to support motivation and recruitment to mathematics, and (d) technological support for teaching processes_-such as digital task assignment and marking.

\section{Technologies Used for Mathematics Instruction}

Computer Algebra Systems, Dynamic Geometry Systems and spreadsheets has been a part of mathematics instruction for decades, yet the mediations of the technologies as well as the research problems addressed by the community is still developing. The presentations in the TSG showed that these technologies are to some extend adopted in the mathematics education practices. The contributions relating to these technologies hence addressed issues relating to teachers adoption, the possibility to deploy such technologies for supporting low achievers, the teaching of specific mathematical concepts in new ways with technology, and the integration of these technologies into learning management systems. Learning management systems signifies a class of systems that is used to support and augment teaching practices. In relation to these systems a number of initiatives to augment their mathematical capabilities were presented. Apart from the integration of Computer Algebra Systems and Dynamic Geometry Software into learning management systems, the work related to the use of such systems related to the construction of multimodal learning environments including video and interactive manipulatives, within learning management systems. Technology that allows for the development of interactive visualizations and for sharing content were presented for several topics and educational levels ranging from primary school to university. Online task environments for students to train their skills with mathematical tasks were also presented. Online streaming of video was presented both as stand-alone and as a part of an online environment for teaching of mathematics. One project applying tablet pcs and interactive whiteboards was also presented. Hence a wide range of the applicable educational technologies where present in the Topic Study Group. 


\section{Problems Addressed in the TSG}

The main problem addressed in the contributions was the potentials of using technology to enhance teaching of mathematics to become a more efficient enterprise. This problem was addressed in a multitude of ways in the topic study group. Interventions aiming at using technology (typically CAS and DGS) to teach mathematical topics in new ways where presented in the groups. It is difficult to summarize the role and influence of technology across these interventions since many factors other than the use of technology influences such interventions. Teacher training and teacher practice with technology conducted was addressed in several of the contributions. One motivation for a specific attendance to this area is that the teachers' choices and practices are, in many ways determining for the success of technology integration in the teaching of mathematics. Motivation and recruitment is an important theme underlying several of the contributions to the topic study group. New interactive illustrations or video presentations might not only make it possible for more students to grasp the abstract mathematical concepts, it might also make mathematics more appealing to larger groups of students.

Apart from addressing concerns, some of the reports in the Topic Study Group also demonstrated new technological developments, addressing the aim of improving mathematics instruction. Technology can automate aspects of the process of teaching mathematics, such as assigning and marking tasks. This is a development with many possible advantages and represents an area were the technological development currently is quite rapid. Another area where new technological developments were presented was the integration of mathematical tools such as CAS and DGS, into web 2.0 internet technology, in a way that supports collaboration and distance education.

A special issue of the electronic Journal of Mathematics and Technology is under preparation. This issue will include papers from the Topic Study Group.

Open Access This chapter is distributed under the terms of the Creative Commons Attribution Noncommercial License, which permits any noncommercial use, distribution, and reproduction in any medium, provided the original author(s) and source are credited. 